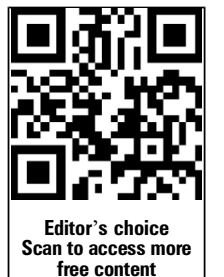

- Additional material is published online only. To view please visit the journal online (http://dx.doi.org/10.1136/ jclinpath-2014-202173).

${ }^{1}$ Translational Cancer Pathology Laboratory, School of Pathology and Laboratory Medicine, University of Western Australia, Crawley, Western Australia, Australia ${ }^{2}$ Cure Brain Cancer Neurooncology Group, Prince of Wales Clinical School, UNSW Australia

${ }^{3}$ Department of Medical Oncology, School of Medicine and Pharmacology, University of Western Australia, Sir Charles Gairdner Hospital, Nedlands, Western Australia, Australia

\section{Correspondence to} Dr Kerrie L McDonald, Cure Brain Cancer Neuro-oncology Group, Prince of Wales Clinical School, Level 2, Lowy Cancer Research Centre, UNSW, Kensington, New South Wales 2052, Australia; k.mcdonald@ unsw.edu.au

AGOG Network Authors are listed in online supplementary table $\mathbf{S 1}$

Received 8 January 2014 Accepted 3 March 2014 Published Online First 2 April 2014

\title{
Multigene profiling to identify alternative treatment options for glioblastoma: a pilot study
}

\author{
Tania Tabone, ${ }^{1}$ Hazem J Abuhusain, ${ }^{2}$ Anna K Nowak, ${ }^{3}$ Australian Genomics and \\ Clinical Outcome of Glioma (AGOG) Network, Wendy N Erber, ${ }^{1}$ Kerrie L McDonald ${ }^{2}$
}

\section{ABSTRACT}

Glioblastoma (GBM) is a highly aggressive malignancy and the most effective treatment regime has a high relapse rate. Increasingly, the development of therapies involves defining drug-diagnostic combinations where the presence of a molecular target or marker identifies patients who are most likely to respond to a specific therapy. Trials in other solid cancers have demonstrated clear utility in the incorporation of biomarkers to stratify patients to targeted treatment, however, there are no mutations that are currently used to inform treatment options for GBM.

Aims We piloted the use of high-throughput nextgeneration sequencing technology to identify genetic mutations in 44 GBM specimens that may be amenable to current or future targeted therapeutic strategies. Method Somatic mutation profiling was performed using the AmpliSeq Cancer Hotspot Panel v2 and semiconductor sequencing technology.

Results A total of 66 mutations were detected in $35 /$ $44(80 \%)$ patients. The number of mutations per tumour ranged from 0 to 4 (average per tumour $=1.5$ ). The most frequent mutations were in TP53 $(n=12)$, PTEN $(n=9)$, EGFR $(n=8)$ and PIK3CA $(n=5)$. Clinically actionable somatic mutations were detected in 24/35 (69\%) patients.

Conclusions This study demonstrates that the use of an 'off-the-shelf' oncogene primer panel and benchtop next-generation sequencer can identify mutations and potentially actionable targets in the majority of GBM patients. Data from this pilot highlights the potential for targeted genetic resequencing to identify mutations that may inform treatment options and predict outcomes.

\section{INTRODUCTION}

Identification of somatically acquired genetic mutations has proven useful in unravelling the pathogenesis of many cancers. Genetic mutations also serve as biomarkers for disease diagnosis, classification and stratification. Advances in throughput and sensitivity of mutation screening technologies now provide a platform to implement biomarkers to assess disease prognosis, identify therapeutic targets and monitor treatment response through detection of minimal residual disease.

Glioblastoma (GBM) is not preventable by any known lifestyle changes, and there has been no significant change in the 5 -year relative survival $(<5 \%)$ in over 20 years. There is currently no curative treatment for GBM and progression is usually rapid and aggressive, hence, this form of cancer is fatal and presents a significant burden in terms of years of life lost. GBM is particularly heterogeneous, and as a consequence, each therapeutic is only likely to be effective in a small proportion of patients. It has never been more clinically relevant to identify somatically acquired mutations in the hope of better matching therapeutic options available for patients with advanced disease.

The Cancer Genome Atlas (TCGA) has generated an unprecedented amount of multidimensional data and is providing a global description of the genetic abnormalities that are present in GBM. An average of 47 mutations per GBM have been identified, although far fewer were candidates to be driver mutations. ${ }^{1}$ Candidate driver mutations are most frequent in the TP53 pathway, RB1 pathway, and the PI3K/PTEN pathway. Mutations in these pathways are generally mutually exclusive, suggesting that they are key to tumourigenesis, and functionally equivalent. ${ }^{2}$ Several genetic alterations operative in the development of GBM have been considered prognostic, including amplification of EGFR, ${ }^{3-6}$ TP53 mutations $^{17}$ and PTEN mutations. ${ }^{8}{ }^{9}$ At least two distinct cluster profiles have been identified: proneural and mesenchymal-angiogenic signatures, which differ in survival and response to treatment. ${ }^{10}{ }^{11}$ Proneural tumours are more commonly IDH1 mutated, and a better prognostic group. ${ }^{12} 13$

The scale of sequencing undertaken by the TCGA could never be replicated for every patient's tumour in the domestic pathology laboratory. However, targeted resequencing of cancer consensus genes has potential for implementation in a routine clinical setting. This method is rapid and cost effective compared to multiple individual diagnostic tests, and the data output is bioinformatically manageable compared to whole genome sequencing approaches. Multigene mutational profiling proves a wealth of information to the treating clinician to identify the best treatment options for individual patients, so that these can be administered in a timely manner for better outcome. Herein, we screened DNA from 44 GBM patients for somatic mutations in 50 oncogenes and tumour suppressor genes using semiconductor sequencing.

\section{MATERIALS AND METHODS \\ Cohort}

Fresh-frozen GBM tumour samples $(n=44)$ were identified for this study from the prospectively collected Australian Genomics and Clinical Outcome of Glioma (AGOG) Biospecimen Resource. Human Research Ethics Committee approval was obtained for the collection and use of freshly frozen human GBM tissue for this project; all participants had previously provided written informed consent for
Abuhusain HJ, Nowak AK,

et al. I Clin Pathol

2014;67:550-555 
tissue biobanking and research use. Only newly diagnosed, untreated GBM specimens were included in this study. Patients were selected based on a diagnosis of primary (de novo) GBM and to incorporate a range of common survival outcomes from $<6$ months to $>24$ months. Patient age, gender, tumour location and other genetic markers (ie, MGMT methylation status and EGFR amplification) were randomised across the survival groups. A summary of the clinical features for each tumour is provided in table 1 . No germline DNA was available for this study. All patients were IDH1 wild type at position 132 according to previous immunohistochemical (IHC) study data. DNA from $50 \mathrm{mg}$ of snap-frozen tissue was extracted and purified using the QIAamp DNA Mini Kit as per protocol (Qiagen). The purified DNA was quantified using the Qubit 2.0 Fluorometer and Qubit dsDNA HS Assay Kit (Life Technologies).

\section{MGMT methylation analysis}

Bisulfite modification followed by $\mathrm{CpG}$ pyrosequencing was performed to assess the percentage level of MGMT promoter methylation for each tumour specimen. Chemically methylated or unmethylated universal human genomic DNA (Millipore) controls were included. In brief, tumour DNA (500 ng) was bisulfite modified using the EZ DNA methylation kit (Zymo Research) according to the manufacturer's recommendations. The CpG pyrosequencing methylation assay was performed with the PyroMark MGMT kit (Qiagen) on a pyrosequencer (PSQ96 MA System, Qiagen) MA system (Qiagen), according to the manufacturer's protocol and as published previously. ${ }^{14}$

\section{Detection of EGFR gene amplification}

EGFR amplification was reported in the patient pathology reports by PathWest Laboratory Medicine WA, using the
Multiplex Ligation-dependent Probe Amplification (MLPA, MCR-Holland) assay as described previously. ${ }^{15}$

\section{AmpliSeq Library preparation}

Amplicon libraries for individual patient DNA samples were prepared using the Ion AmpliSeq Ready-to-use Panel to amplify the target regions of 50 oncogenes and tumour suppressor genes implicated in solid tumours (see online supplementary table S2). Target regions were amplified from $10 \mathrm{ng}$ of genomic DNA in a single multiplex PCR reaction using the premixed Ion AmpliSeq Cancer HotSpot Panel v2 and the AmpliSeq HiFi Master Mix (Ion AmpliSeq Library Kit 2.0). The resulting 207 multiplexed amplicons were treated with FuPa Reagent (Life Technologies) to partially digest the primer sequences and phosphorylate the amplicon ends. Sequencing adapters with unique barcodes (Ion Xpress Barcode Adapters 1-96, Life Technologies) were ligated to the amplification products and purified using Agencourt AMPure XP Reagent (Beckman Coulter) according to the manufacturer's instructions. The amplicon libraries underwent a second round of amplification to quantify and visualise the library fragments using the Agilent 2100 Bioanalyser and Agilent High Sensitivity DNA Kit (Agilent Technologies) according to the manufacturer's instructions. The final library concentrations were standardised to $100 \mathrm{pM}$ in low Tris-EDTA (TE) buffer (Life Technologies).

\section{Emulsion PCR and semiconductor sequencing}

Eight individual patient barcoded libraries (100pM each) were pooled, and the final concentration adjusted to $9 \mathrm{pM}$ in nuclease-free water. Multiplexed barcoded libraries were then clonally amplified with biotinylated primers onto Ion Sphere Particles (ISPs; Ion Xpress Template Kit 2.0) by emulsion PCR

Table 1 Clinical data for GBM patients divided into specific survival times

\begin{tabular}{|c|c|c|c|c|}
\hline & $\begin{array}{l}\text { Survival group } 1 \\
<6 \text { months }\end{array}$ & $\begin{array}{l}\text { Survival group } 2 \\
6-12 \text { months }\end{array}$ & $\begin{array}{l}\text { Survival group } 3 \\
12-24 \text { months }\end{array}$ & $\begin{array}{l}\text { Survival group } 4 \\
>24 \text { months }\end{array}$ \\
\hline Patient numbers & 8 & 13 & 15 & 8 \\
\hline Median survival (months) $(95 \% \mathrm{Cl}$ ) & 4.0 (3.7 to 4.1$)$ & $10.5(7.2$ to 13.9$)$ & 14.2 (13.8 to 14.6$)$ & 30.3 (27.7 to 32.9$)$ \\
\hline Median age (years) (range) & $66(51-85)$ & $65(38-76)$ & $60(45-77)$ & $59(24-70)$ \\
\hline \multicolumn{5}{|l|}{ Location in the brain } \\
\hline Frontal & 3 & 6 & 2 & 4 \\
\hline Temporal & 3 & 4 & 7 & 2 \\
\hline Parietal & - & 3 & 3 & 1 \\
\hline Occipital & 1 & - & 1 & 1 \\
\hline Multifocal & - & - & 1 & - \\
\hline Unknown & 1 & - & 1 & - \\
\hline \multicolumn{5}{|l|}{ Extent of surgery } \\
\hline Total resection & 8 & 12 & 14 & 8 \\
\hline Biopsy & 0 & 1 & 1 & 0 \\
\hline \multicolumn{5}{|l|}{ Received concurrent treatment } \\
\hline Yes & 3 & 12 & 15 & 8 \\
\hline No & 5 & 1 & 0 & 0 \\
\hline \multicolumn{5}{|l|}{ MGMT promoter methylation } \\
\hline Yes & 2 & 1 & 3 & 3 \\
\hline No & 6 & 11 & 9 & 2 \\
\hline Not tested & 0 & 1 & 3 & 3 \\
\hline \multicolumn{5}{|l|}{ EGFR amplification } \\
\hline Yes & 1 & 2 & 4 & 1 \\
\hline No & 2 & 2 & 3 & 2 \\
\hline Not tested & 5 & 9 & 8 & 5 \\
\hline
\end{tabular}


(emPCR) using the OneTouch 2 System (Life Technologies). Following emPCR, the ISPs were recovered by centrifugation and template-positive ISPs were isolated by binding to streptavidin-coated beads (Ion OneTouch 2 Kit). A sequencing primer was ligated to the enriched ISPs before loading onto an Ion 316 Chip for single-end sequence analysis. Semiconductor sequencing was performed on an Ion Personal Genome Machine (PGM) Sequencer using the Ion PGM 200 Sequencing Kit (Life Technologies).

\section{Coverage and data analysis}

Torrent Suite V.3.6.2 Software (Life Technologies) was used to parse barcoded reads, align reads to the reference genome (human genome build 19; hg19), base call and to generate run metrics, including chip loading efficiency, total read counts, quality and total coverage. ANOVAR (Biobase) and Oncomine Gene Browser (Compendia) were used to identify variants and predict amino acid changes and clinical significance. The Integrative Genomics Viewer (IGV) was used to visualise the read alignment and the presence of variants against the reference genome and to confirm the integrity of variant calls by detecting possible strand bias and sequencing errors.

\section{RESULTS}

\section{Clinicopathological correlation}

The study population consisted of 44 GBM patients with a higher ratio of males to females $(30: 14)$. The median age at diagnosis was 63.3 years (range $24-85$ years). The median survival rate was 12.0 months (range $3.7-41.5$ months) (table 1 ). The majority of tumours were localised to the frontal (15 patients, 34\%) and temporal lobes (16 patients, 36\%). Seven patients had GBM arising in the parietal lobe region, three patients had GBM arising in the occipital lobe, and one patient's GBM was multifocal. The tumour location was unknown for two patients. The majority of patients had total (greater than $95 \%$ ) tumour resection during their first surgery and two patients received biopsies only. Following surgery, 38 out of 44 patients received combined radiotherapy and chemotherapy (temozolomide (TMZ)). ${ }^{16}$ MGMT promoter methylation results were available for 37 of the 44 patients. The percentage methylated of those tested was 24\%. EGFR amplification is not routinely requested as a pathology test, thus, results were only available for a small subset of the cohort (19 out of 44). EGFR amplification was detected in almost half the tumours tested (9 out of 19).

The 44 GBM patient cohort was divided into four groups according to survival outcomes: Group $1(n=8)$ : survival $<6$ months; Group $2(n=13)$ : survival $6-12$ months; Group 3 $(n=15)$ : survival $12-24$ months and Group $4(n=8)$ : survival $>24$ months (table 1; figure 1). Age of the patients did not significantly differ between the four groups ( $p$ value: 0.151). A high percentage of patients who did not receive radiotherapy or any adjuvant chemotherapy showed very poor overall survival (Group 1).

\section{Sequence coverage}

Analysis of our sequencing data showed a mean coverage depth of 1600 reads per nucleotide position within the target region. The $1 \times, 10 \times$ and $100 \times$ coverage were $100 \%, 100 \%$ and $99 \%$, respectively. Sequence coverage was assessed from the number and distribution of reads across target amplicons. An average of 5.3 million of the total 6.3 million addressable wells in the Ion 316 Chip were consistently loaded with ISPs, and after subtracting poly clones (multiple-templated beads), low quality sequence reads $(<\mathrm{Q} 20)$ and primer dimer, 3.3 million $(62.2 \%)$ of these particles contained library templates. The individual samples averaged 373784 mapped sequence reads (range 312 271653751 ), with a mean read depth of 1809 per variant. The distribution of reads across the 207 amplicons was consistent across sample, with an average uniformity of amplicon coverage of $95.41 \%$ and $95.92 \%$ of the sequence reads mapped to targeted gene regions (aligned to hg19). The mean read length was 111 bp (range 105-114 bp).

\section{Sequence validation}

A minimum of 300000 reads with a quality score of AQ20 (one misaligned base per 100 bases) was used as the measure of successful sequencing of a sample. For a variant to be considered true, sequencing coverage of $1000 \times$ and a variant frequency of at least $10 \%$ in a background of wild-type allele were used as a minimum requirement in this study.

\section{Variants detected}

Overall, 17288299 nucleotides were interrogated across the 44 GBM cases analysed. A total of 644 mutations in 32 genes were identified, with a mean of 14 variants per patient (range 7-21 variants). Since constitutional DNA was not available to deduce germline polymorphisms, a stringent mutation detection criterion was applied in order to identify somatically acquired mutations. Mutations present in the population with a minor allele frequency greater than 5\% according to the 1000 Genomes Project, intronic mutations and synonymous exonic mutations were removed. Therefore, a total of 66 variants were predicted to cause non-synonymous coding changes in 18 different genes, with an average of 1-2 variants detected per patient (range 0-4 variants) (table 2; figure 1). Of these, 48 variants were unique in the study cohort with 39 variants not previously described in dbSNP, 1000 Genomes Project or the published literature. Using this stringent mutation detection approach, there were nine GBM cases in which no significant mutations were detected.

The non-synonymous mutations detected are summarised in online supplementary table S3 and graphically represented in figure 1. In the poorest survival group (Group 1: survival $<6$ months, $\mathrm{n}=8$ ), two patient tumours' harboured no nonsynonymous mutations. For the remaining patients in Group 1, the most commonly mutated gene was PTEN found in two out of the six patients, however, the mutation position differed. Patient 17 harboured 2 PTEN mutations (p.Q171R and p. F241S), while patient 15 had a stop-gain mutation (p.R233X). Five of the six patients harboured potentially treatable mutations. Two patients were methylated at the MGMT promoter, however, these two individuals were not treated with temozolomide.

For the Group 2 patients (survival 6-12 months, $n=13$ ), three patients harboured multiple mutations $(\geq 2)$. The EGFR mutation, p.A289 V, located in the extracellular domain (ED) region of EGFR was detected in four patients. Eighty-two per cent of the mutations detected in Group 2 were potentially amenable to drugs, which is clinically interesting given that the majority of the patients (92\%) were unmethylated at the MGMT promoter. Patients belonging to group 3 demonstrated a higher survival time (survival $12-24$ months, $n=15$ ) compared to the cohort survival median time (12.0 months (range 3.741.5 months). Forty-seven per cent of the tumours were located in the temporal region of the brain (7 out of 15). Multiple nonsynonymous mutations $(\geq 2)$ were seen in six patients. Mutations in TP53 were common (50\%). Mutations in the 

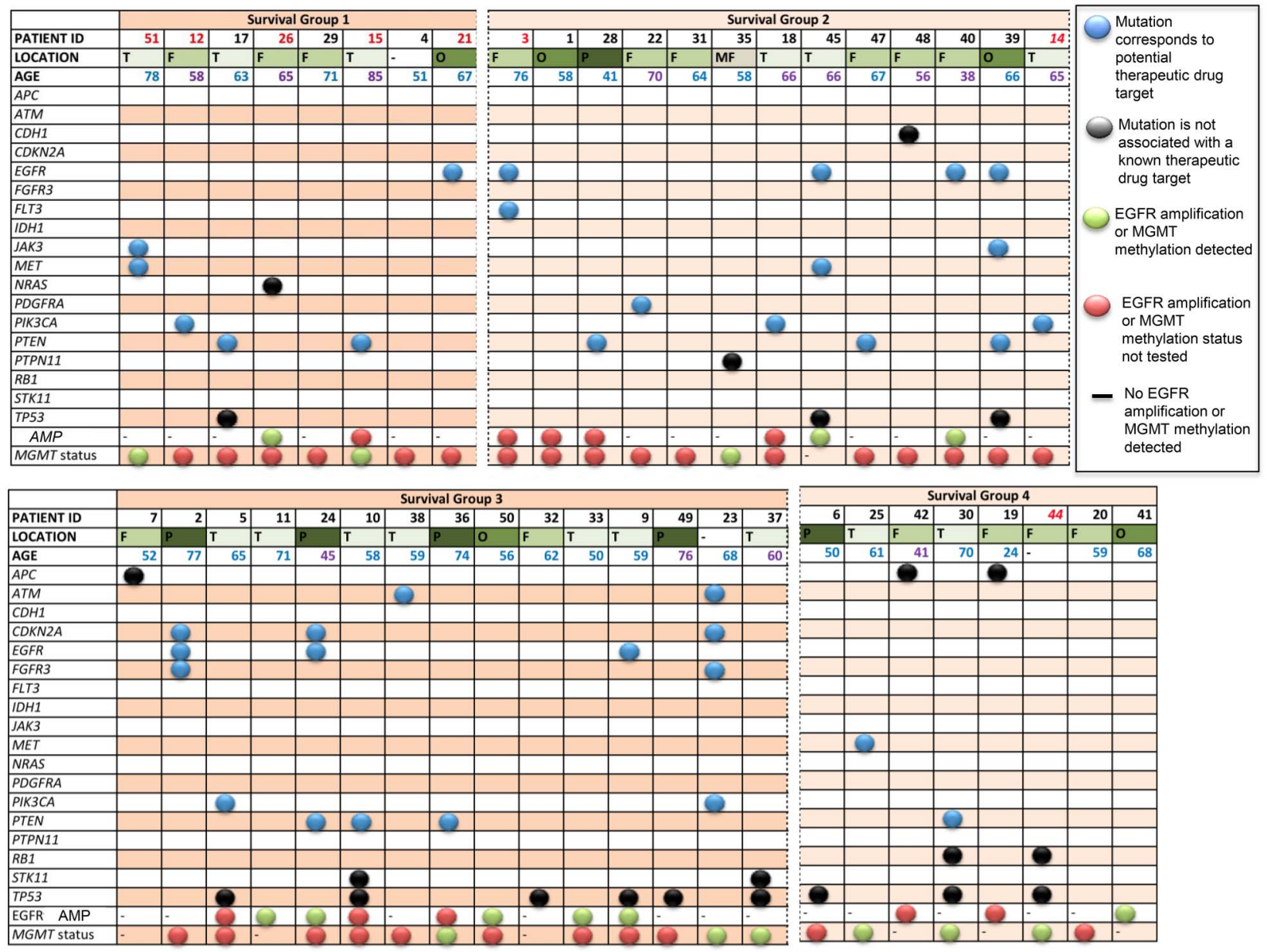

Figure 1 Distribution of mutations in patients categorised by overall survival times. The mutational profile of 18 genes is illustrated. Patients are listed in order of increasing time of survival and grouped into 4 survival groups: Group 1: survival $<6$ months; Group 2: survival 6-12 months; Group 3: survival 12-24 months and Group 4: >24 months. Blue spot: mutation corresponding to a potential therapeutic drug target; Black spot: mutation is not associated with a known therapeutic drug target; Green spot: EGFR amplification or MGMT methylation detected; Red spot: no EGFR amplification or MGMT methylation detected; (-) represents EGFR amplification or MGMT methylation status not tested. Tumour location is indicated at the top of the figure where: $T$, temporal; $F$, frontal; 0 , occipital; $\mathrm{P}$, parietal; MF, multifocal. Age is given in years and the blue font represents male and the purple font represents female.

EGFR gene were also detected, and although localised to the $\mathrm{ED}$, they were all located in different positions (p.G392V, p. R108K and one tumour had both p. A289V and p.V729M mutations). Amplification of the EGFR gene was also a common event in this group with five of the eight tested showing amplification. Three patients had PTEN mutations, while two had mutations in CDKN2 and STK11. Promoter methylation of the MGMT gene was again uncommon (25\%). Sixty-seven per cent of patients harboured mutations that were potentially treatable with targeted agents.

In the longest survival cohort, Group 4 ( $>24$ months, $n=8)$, no mutations were detected in two of the patients. Mutations in the TP53 gene were detected in four out six patients. Two patients harboured mutations in $R B 1$ and another two patients had mutations in the APC gene. These mutations were not present in the patients belonging to the other three survival groups. Unfortunately, there are no known therapeutics to target TP53, RB1 and APC. Therefore, four out of the six patients in this survival group would not benefit from genetargeted therapy. Incidentally, MGMT promoter methylation was detected in three out five of the tested patients.

\section{DISCUSSION}

Diagnosis and management of many solid tumours now relies on the integration of genomic information into the routine pathology workflow and the consolidation of a range of testing to a single platform is desirable. For a complex disease such as GBM, a single platform and a single assay may never be achieved because of the different testing modalities needed to detect methylation, chromosomal loss, gene amplification and deletion and protein expression. However, mutations in key genes such as TP53, EGFR, PTEN and IDH1 are not currently being tested for in routine pathology, and the field would significantly benefit from further development of multigene mutational profiling.

A significant unmet need is to discover new treatments to improve the standard-of-care therapy (chemoradiotherapy: radiation therapy plus temozolomide followed by six cycles of adjuvant temozolomide). MGMT promoter methylation is a strong predictor of benefit with chemoradiotherapy. ${ }^{17-20}$ This predictive role was prospectively confirmed by the Radiation Therapy Oncology Group (RTOG) study 0525. ${ }^{21}$ MGMT promoter methylation status is also a useful predictive marker for benefit 
Table 2 Summary of sequencing data

\begin{tabular}{lr}
\hline Total number of genes mutated & 32 \\
Total number of mutations: & 644 \\
UTR3 & 87 \\
Intronic & 89 \\
Splicing & 46 \\
ncRNA, intronic & 3 \\
Exonic, synonymous & 279 \\
Exonic, non-synonymous & 138 \\
Exonic, frameshift & 3 \\
Exonic, stopgain & 5 \\
Exonic, stoploss & 1 \\
Total number of genes mutated with a MAF <5\% & 24 \\
Total number of mutations with a MAF <5\%: & 113 \\
UTR3 & 2 \\
Intronic & 30 \\
Exonic, synonymous & 15 \\
Exonic, non-synonymous & 58 \\
Exonic, frameshift & 2 \\
Exonic, stopgain & 5 \\
Exonic, stoploss & 1 \\
Total number of genes with a non-synonymous mutation & 17 \\
Total number of non-synonymous mutations & 66 \\
\hline
\end{tabular}

MAF, minor allele frequency; ncRNA, non-coding RNA; UTR, untranslated region. sensitivity to erlotinib in patients harbouring ED mutations. ${ }^{26}$ Furthermore, targeted therapy has been used effectively for patients with non-small cell lung cancer (NSCLC) with responsiveness to the small molecule EGFR tyrosine kinase inhibitors (TKIs), erlotinib and gefitinib, tightly linked to the presence of missense mutations in the EGFR kinase domain. While current clinical trials with these TKIs have failed to show any response in GBM clinical trials, the presence or absence of mutations in EGFR were not taken into consideration in these studies. ${ }^{27} \mathrm{~A}$ restored hope for targeted EGFR therapy for GBM has been generated from the development of second-generation irreversible EGFR inhibitors such as dacomitinib (PF-00299804). ${ }^{29}$ Dacomitinib has a higher affinity for the EGFR ED and irreversible blockade may result in a longer suppression of EGFR.

Mutations in the TP53 gene are more commonly associated with low-grade astrocytomas (50-60\%) and secondary GBM $(70 \%) .{ }^{30}$ However, similar to the TCGA analysis of $\mathrm{GBM},{ }^{1}$ we also found a high incidence of TP53 in our GBM cohort. Thirty-eight per cent of the mutations found resided within the DNA binding domain (exons 4-8) of TP53. Missense mutations in this region, but not the transactivation domain (exons 1-3) or the oligomerisation domain (exons 9-11), influence the survival outcomes of patients with breast cancer. ${ }^{31}$ Sequencing of TP53 is not carried out in neuropathology. Rather, IHC detection of the mutant TP53 protein, based on a higher percentage staining reflective of the mutant protein's longer half-life, is conducted. It is not accurate and is not clinically relevant.

In summary, this pilot study of 44 de novo GBM demonstrates that the use of an 'off-the-shelf' oncogene primer panel and benchtop next-generation sequencer can identify mutations and potentially actionable targets in the majority of GBM patients. Although not shown here, the methodology is applicable to formalin-fixed paraffin-embedded tissue and, as such, it could be adopted into a clinical setting to accompany other routine tests. Further, the mutation yield could be further improved with a less 'cancer-generic' and more specific GBM primer

\section{Take home messages}

- Studies and trials in other solid cancers, including breast, pancreatic and non-small cell lung cancer (NSCLC) have demonstrated clear usefulness in drug-diagnostic combinations, however, there are no mutations that are currently used to inform treatment options for glioblastoma (GBM).

- GBM is heterogeneous and as a consequence, each therapeutic is only likely to be effective in a small proportion of patients.

- The sequencing of clinically actionable somatic mutations in GBM is highly relevant, but not conducted. This single platform assay could be developed preclinically with a view to be used clinically to inform drug trials exploring targeted agents to EGFR, PTEN, PIK3CA and CDK. Additionally, mutations in the TP53 and RB1 genes may influence response to a wide range of drugs targeted to the cell cycle of the cancer.

- Data from this pilot study highlights the potential for targeted genetic resequencing to identify mutations that may inform treatment options. Future large-scale trials will be required to validate and determine the true clinical utility of this approach for implementation into the clinic. tumours in vivo demonstrating that the EGFR ED mutants are oncogenic. $^{26}$ The same study also demonstrated a greater mutations were all localised to the ED region. ${ }^{25}$ The most common mutation identified was A289 V (4 out of 8 GBM) and single tumours with the mutation R108K and G598V. One study has reported that the ectopic expression of these mutations (A289V, R108K and G598V) in NIH-3T3 fibroblasts con-
ferred anchorage-independent colony formation, and formed 
panel. There is an urgent need to find alternative therapies for MGMT unmethylated GBM and the development of therapies will no doubt involve defining drug-diagnostic combinations where the presence of a molecular target or marker identifies patients who are most likely to respond to a specific therapy.

This pilot study highlights the potential for targeted genetic resequencing to identify mutations that may inform treatment options. To complement this technology, patient-derived cell lines harbouring the same mutations would provide a valuable resource for high throughput testing of targeted drugs with a broad range of anti-tumour activity. Accurate diagnosis, which includes traditional histopathology and molecular histology, will lead to better treatment selection and improved survival times. Future large-scale studies will be required to determine the clinical usefulness of this approach for screening or monitoring purposes.

Acknowledgements For technical support and expertise we thank R Allcock.

Collaborators Australian Genomics and Clinical Outcomes of Glioma (AGOG) Network.

Contributors TT performed experimental work and data analysis. HJA and AN provided patient materials. WNE provided project leadership. KLM collected clinical data and patient materials and coordinated the study. All authors contributed to the final manuscript.

Funding This work was principally supported by the Olle Fund For Brain Cancer Research. This work was also supported by the Cancer Council NSW with assistance from Cancer Australia.

Competing interests TT was supported by a Cancer Pathology Fellowship from Cancer Council Western Australia. KLM was supported by the Cure Brain Cancer Foundation.

Ethics approval Human Research Ethics Committee South Eastern Sydney Local Health District Prince of Wales Hospital.

Provenance and peer review Not commissioned; externally peer reviewed.

\section{REFERENCES}

1 Cancer Genome Atlas Research Network. Comprehensive genomic characterization defines human glioblastoma genes and core pathways. Nature 2008:455:1061-8.

2 Kleihues P, Louis DN, Scheithauer BW, et al. The WHO classification of tumours of the nervous system. J Neuropathol Exp Neurol 2002;61:215-25; discussion 226219.

3 Toth J, Egervari K, Klekner A, et al. Analysis of EGFR gene amplification, protein over-expression and tyrosine kinase domain mutation in recurrent glioblastoma. Pathol Oncol Res 2009;15:225-9.

4 Viana-Pereira M, Lopes JM, Little S, et al. Analysis of EGFR overexpression, EGFR gene amplification and the EGFRvIll mutation in Portuguese high-grade gliomas. Anticancer Res 2008:28(2A):913-20.

5 Shinojima N, Tada K, Shiraishi S, et al. Prognostic value of epidermal growth factor receptor in patients with glioblastoma multiforme. Cancer Res 2003;63:6962-70.

6 Layfield LJ, Willmore C, Tripp S, et al. Epidermal growth factor receptor gene amplification and protein expression in glioblastoma multiforme: prognostic significance and relationship to other prognostic factors. Appl Immunohistochem Mol Morphol 2006;14:91-6.

7 Olar A, Aldape KD. Biomarkers classification and therapeutic decision-making for malignant gliomas. Curr Treat Options Oncol 2012;13:417-36.

8 Hill C, Hunter SB, Brat DJ. Genetic markers in glioblastoma: prognostic significance and future therapeutic implications. Adv Anat Pathol 2003;10:212-17.
9 Kraus JA, Glesmann N, Beck M, et al. Molecular analysis of the PTEN, TP53 and CDKN2A tumour suppressor genes in long-term survivors of glioblastoma multiforme. J Neurooncol 2000:48:89-94.

10 Colman $\mathrm{H}$, Zhang L, Sulman EP, et al. A multigene predictor of outcome in glioblastoma. Neuro Oncol 2010;12:49-57.

11 Verhaak RG, Hoadley KA, Purdom E, et al. Integrated genomic analysis identifies clinically relevant subtypes of glioblastoma characterised by abnormalities in PDGFRA, IDH1, EGFR, and NF1. Cancer Cell 2010;17:98-110.

12 Parsons DW, Jones $S$, Zhang $X$, et al. An integrated genomic analysis of human glioblastoma multiforme. Science 2008;321:1807-12.

13 Turcan S, Rohle D, Goenka A, et al. IDH1 mutation is sufficient to establish the glioma hypermethylator phenotype. Nature 2012;483:479-83.

14 McDonald KL, Rapkins RW, Olivier J, et al. The T genotype of the MGMT C>T (rs16906252) enhancer single-nucleotide polymorphism (SNP) is associated with promoter methylation and longer survival in glioblastoma patients. Eur J Cancer 2013:49:360-8

15 Jeuken J, Sijben A, Alenda C, et al. Robust detection of EGFR copy number changes and EGFR variant III: technical aspects and relevance for glioma diagnostics. Brain Pathol 2009:19:661-71.

16 Stupp R, Mason WP, van den Bent MJ, et al. Radiotherapy plus concomitant and adjuvant temozolomide for glioblastoma. N Engl J Med 2005:352:987-96.

17 Gallego Perez-Larraya J, Ducray F, Chinot O, et al. Temozolomide in elderly patients with newly diagnosed glioblastoma and poor performance status: an ANOCEF phase II trial. J Clin Oncol 2011;29:3050-5.

18 Wick W, Platten M, Meisner C, et al. Temozolomide chemotherapy alone versus radiotherapy alone for malignant astrocytoma in the elderly: the NOA-08 randomised, phase 3 trial. Lancet Oncol 2012;13:707-15.

19 Brandes AA, Franceschi E, Tosoni A, et al. Temozolomide concomitant and adjuvant to radiotherapy in elderly patients with glioblastoma: correlation with MGMT promoter methylation status. Cancer 2009;115:3512-18.

20 Hegi ME, Diserens AC, Gorlia T, et al. MGMT gene silencing and benefit from temozolomide in glioblastoma. N Engl J Med 2005:352:997-1003.

21 Ahluwalia MS. American Society of Clinical Oncology 2011 CNS tumours update. Expert Rev Anticancer Ther 2011:11:1495-7.

22 Malmstrom A, Gronberg BH, Marosi C, et al. Temozolomide versus standard 6-week radiotherapy versus hypofractionated radiotherapy in patients older than 60 years with glioblastoma: the Nordic randomised, phase 3 trial. Lancet Oncol 2012;13:916-26

23 Quinn JA, Jiang SX, Reardon DA, et al. Phase II trial of temozolomide (TMZ) plus irinotecan (CPT-11) in adults with newly diagnosed glioblastoma multiforme before radiotherapy. J Neurooncol 2009;95:393-400.

24 Ciriello G, Miller ML, Aksoy BA, et al. Emerging landscape of oncogenic signatures across human cancers. Nat Genet 2013;45:1127-33.

25 Idbaih A, Aimard J, Boisselier B, et al. Epidermal growth factor receptor extracellula domain mutations in primary glioblastoma. Neuropathol App/ Neurobiol 2009;35:208-13.

26 Lee JC, Vivanco I, Beroukhim R, et al. Epidermal growth factor receptor activation in glioblastoma through novel missense mutations in the extracellular domain. PLOS Med 2006:3:e485.

27 Cho J, Pastorino S, Zeng Q, et al. Glioblastoma-derived epidermal growth factor receptor carboxyl-terminal deletion mutants are transforming and are sensitive to EGFR-directed therapies. Cancer Res 2011;71:7587-96.

28 Haas-Kogan DA, Prados MD, Lamborn KR, et al. Biomarkers to predict response to epidermal growth factor receptor inhibitors. Cell Cycle 2005:4:1369-72.

29 Ather F, Hamidi H, Fejzo MS, et al. Dacomitinib, an irreversible Pan-ErbB inhibitor significantly abrogates growth in head and neck cancer models that exhibit low response to cetuximab. PloS ONE 2013;8:e56112.

30 Ohgaki $\mathrm{H}$, Kleihues P. Genetic alterations and signaling pathways in the evolution of gliomas. Cancer Sci 2009;100:2235-41.

31 Vegran $F$, Rebucci $M$, Chevrier $S$, et al. Only missense mutations affecting the DNA binding domain of p53 influence outcomes in patients with breast carcinoma. PloS ONE 2013;8:e55103. 